\title{
Chondroprotective Effect of Kartogenin on CD44-Mediated Functions in Articular Cartilage and Chondrocytes
}

Cartilage

2014, Vol. 5(3) 172-180

(C) The Author(s) 2014

Reprints and permissions:

sagepub.com/journalsPermissions.nav

DOI: 10.1 I77/19476035/4528354

cart.sagepub.com

\author{
Yohei Ono ${ }^{1,2}$, Shinya Ishizuka ${ }^{1,2}$, Cheryl B. Knudson ${ }^{2}$, and Warren Knudson ${ }^{2}$
}

\begin{abstract}
Objective: A recent report identified the small molecule kartogenin as a chondrogenic and chondroprotective agent. Since changes in hyaluronan metabolism occur during cartilage degeneration in osteoarthritis, we began studies to determine whether there was a connection between extracellular hyaluronan, CD44-hyaluronan interactions and the effects of kartogenin on articular chondrocytes. Methods: Chondrocytes cultured in monolayers, bioengineered neocartilages, or cartilage explants were treated with kartogenin with or without stimulation by IL-I $\beta$. Accumulation of matrix was visualized by a particle exclusion assay or by safranin $O$ staining and release of sulfated glycosaminoglycans was determined. Production of aggrecanases and aggrecan GI-ITEGE neoepitope, fragmentation of CD44 and the SMADI/5/8 signaling pathway were evaluated by western blotting. Results: Kartogenin treatment enhanced chondrocyte pericellular matrix assembly and retention in the presence of IL-I $\beta$. The chondroprotective effects of kartogenin on IL-I $\beta$-induced release of sulfated glycosaminoglycans from articular cartilage explants, reduction in safranin $O$ staining of neocartilage discs as well as a reduction in aggrecan GI-ITEGE neoepitope in chondrocyte and explant cartilage cultures were observed. Kartogenin partially blocked the IL-I $\beta$-induced increased expression of ADAMTS-5. Additionally, kartogenin-treated articular chondrocytes exhibited a decrease in CD44 proteolytic fragmentation. However, kartogenin treatment did not enhance proteoglycan in control, non-IL-I $\beta$-treated cultures. Similarly, kartogenin enhanced the SMADI phosphorylation but only following pretreatment with IL-I $\beta$. Conclusion: These studies provide novel information on the chondroprotective function of kartogenin in adult articular cartilage. The effects of kartogenin are significant after activation of chondrocytic chondrolysis, which may occur following disruption of homeostasis maintained by hyaluronan-CD44 interactions.
\end{abstract}

\section{Keywords}

kartogenin, osteoarthritis, chondrocyte, hyaluronan, CD44

\section{Introduction}

One of the early events associated with osteoarthritis is the pronounced loss of aggrecan from the cartilage. ${ }^{1}$ The glycosaminoglycan hyaluronan (HA) is also depleted in tandem with the loss of aggrecan. ${ }^{2-8}$ Aggrecan turnover occurs within the extracellular environment because of the activity of endoproteinases termed aggrecanases as well as matrix metalloproteinases. ${ }^{9,10}$ The aggrecanases ADAMTS4 and ADAMTS5 are thought to be the key mediators of aggrecan loss. ${ }^{11-14}$ Our work has focused on the fate of the remaining portion of the aggrecan aggregate namely, the HA and HA-bound aggrecan-G1 domains terminating with ITEGE $^{373}$ and DIPEN ${ }^{341}$; sequences that are generated following cleavage of aggrecan via aggrecanases or matrix metalloproteinases respectively. Our previous studies have demonstrated that HA and bound G1-ITEGE proteins undergo co-endocytosis by articular chondrocytes, mediated via the HA receptor, CD44. ${ }^{15,16}$
Hyaluronan-CD44 interactions are also the primary means for the retention of aggrecan to the cell surface of chondrocytes. ${ }^{17,18}$ Since HA serves as the core filament of the cartilage proteoglycan aggregate, we hypothesize that the enhancement of HA levels is necessary for chondrocytes to undergo successful cartilage repair such as repair episodes during the early stages of osteoarthritis. Extracellular HA is regulated by the activity of hyaluronan

\footnotetext{
'Department of Orthopaedic Surgery, Nagoya University Graduate School of Medicine, Nagoya, Japan

${ }^{2}$ Department of Anatomy and Cell Biology, The Brody School of Medicine, East Carolina University, Greenville, NC, USA

Corresponding Author:

Warren Knudson, Department of Anatomy and Cell Biology, The Brody School of Medicine, East Carolina University, 600 Moye Boulevard, Greenville, NC 27834, USA.

Email: knudsonw@ecu.edu
} 
synthases (HAS) ${ }^{19}$ and, the local catabolism of hyaluronan by $\mathrm{CD} 44$ receptor-mediated endocytosis. ${ }^{16}$

A recent report identified the small molecule kartogenin as a chondrogenic and chondroprotective agent. ${ }^{20}$ The elevated release of nitric oxide by bovine articular chondrocytes and the release of sulfated glycosaminoglycans from bovine cartilage explants, induced by oncostatin $M$ and $\mathrm{TNF} \alpha$, were significantly reduced on treatment with kartogenin. Also, intra-articular injection of kartogenin reduced tibial plateau cartilage degeneration in the mouse osteoarthritis models. This molecule, kartogenin, is expected to be a possible novel therapeutic drug for the treatment of osteoarthritis. ${ }^{21}$ However, there has not yet been any other published report of kartogenin. Since changes in HA metabolism occur during cartilage degeneration in osteoarthritis, we began studies to determine whether kartogenin exhibited chondroprotective properties in our system. For example, we determined whether kartogenin affected the retention of HA-dependent pericellular matrices as well as other CD44HA interactions that are necessary for the maintenance of articular chondrocytes. In this study, the effects of kartogenin on primary chondrocytes in monolayer cultures, in bioengineered neocartilages, and in cartilage explants, were examined with or without prestimulation with IL-1 $\beta$.

\section{Materials and Methods}

\section{Chondrocyte and Cartilage Explant Culture}

Primary articular chondrocytes were isolated from the metacarpophalangeal joints of 18- to 24-month-old adult steers and from human osteoarthritis knee cartilage following joint replacement within 24 hours after surgery. The acquisition of human cartilage was approved by the Institutional Review Board of East Carolina University. Chondrocytes were liberated from full thickness slices of articular cartilage by sequential pronase/collagenase digestion. ${ }^{22}$ Primary bovine and human chondrocytes were cultured in a DMEM/Ham's F-12 (Corning Cellgro, Manassas, VA) medium containing $10 \%$ fetal bovine serum (FBS, Hyclone, South Logan, UT) and 50 units $/ \mathrm{mL}$ penicillin, L-glutamate, and ascorbic acid. The chondrocytes were plated either as high-density monolayers $\left(1.0 \times 10^{6}\right.$ cells/ $\mathrm{cm}^{2}$ ) for immediate analysis, as low-density monolayers $\left(5,000 \mathrm{cells} / \mathrm{cm}^{2}\right)$ for particle exclusion assay analysis, or cultured in alginate beads as described previously ${ }^{23}$ for subsequent placement into bioengineered neocartilage disks. Chondrocytes released from alginate beads were allowed to form neocartilage by an additional 2 weeks in culture in $0.4-\mu \mathrm{m}$ pore membrane cell culture inserts (Becton Dickinson, Franklin Lakes, NJ) as described previously. ${ }^{23}$ In other experiments, full-thickness cartilage disks were made using a 6-mm biopsy core punch from either bovine articular cartilage or from a relatively intact region on the femoral condyle from an osteoarthritis patient ( 72 years old, male) and cultured as tissue explants.

\section{Treatment of Cells and Explants}

Primary chondrocytes in monolayer or alginate beads, neocartilages or cartilage explants were treated for varying time periods with 1.0 or $10.0 \mathrm{ng} / \mathrm{mL}$ IL- $1 \beta$ (R\&D Systems, Minneapolis, MN) in serum-free culture medium in the presence or absence of 0.1 to $100.0 \mu \mathrm{M}$ kartogenin (Cellagen Technology, San Diego, CA). Dimethylsulfoxide (DMSO) was always used as the carrier control for kartogenin. For analysis of BMP signaling, chondrocytes were treated with $100 \mathrm{ng} / \mathrm{mL} \mathrm{BMP}^{24}$ (R\&D Systems, Minneapolis, MN) or with $10 \mu \mathrm{M}$ kartogenin.

\section{Particle Exclusion Assay}

Particle exclusion assay was performed as described previously. ${ }^{25}$ Briefly, after treatment with IL-1 $\beta$, kartogenin or both, the media of low-density monolayer bovine chondrocytes was replaced with a suspension of formalin-fixed erythrocytes in serum free media. Additionally, the live cells were labeled with calcein AM (Sigma-Aldrich, St. Louis, MO) to delineate the plasma membrane. Cells were photographed using a Nikon TE2000 inverted phase-contrast fluorescence microscope. The presence of cell-bound extracellular matrix was seen as a particle-excluded zone surrounding the chondrocytes. Treatment with 100 units $/ \mathrm{mL}$ testicular hyaluronidase (Sigma-Aldrich) for 1 hour removed the hyaluronan-dependent pericellular coat, whereupon the particles abutted the plasma membrane.

\section{Histology}

After IL-1 $\beta$ and, with or without kartogenin treatment, bovine neocartilage disks were fixed with $4 \%$ buffered paraformaldehyde overnight at $4{ }^{\circ} \mathrm{C}$, rinsed in $30 \%$ sucrose, phosphate-buffered saline, and embedded in OCT-freezing medium. Cryostat sections $(10 \mu \mathrm{m})$ were prepared and stained with safranin $\mathrm{O}$ for the detection of proteoglycans and counterstained with Fast Green.

\section{Determination of Sulfated Glycosaminoglycan Release}

Bovine cartilage explants were pretreated in serum free culture medium in the presence or absence of $100 \mu \mathrm{M}$ kartogenin for 48 hours, and treated with or without IL-1 $\beta$ $(10 \mathrm{ng} / \mathrm{mL})$. The medium samples were collected after 24 hours, and equal volumes were analyzed for sulfated glycosaminoglycans. Shark chondroitin sulfate (Sigma) dissolved at a concentration of 0 to $500 \mu \mathrm{g} / \mathrm{mL}$ in culture 
medium was used to produce a standard curve. Twenty microliters of media were added to a 96-well plate, followed by $180 \mu \mathrm{L}$ of dimethylmethylene blue (DMMB) reagent. After 5 minutes of incubation, absorbance was determined at $525 \mathrm{~nm}$ with a Multiskan Ascent microplate reader. The sulfated glycosaminoglycans content was then calculated from the standard curve. ${ }^{26,27}$

\section{SDS-PAGE and Western Blotting}

For monolayer cultures, cell lysates were prepared using Cell Lysis Buffer (Cell Signaling, Danvers, MA) containing Protease Inhibitor Cocktail (Thermo Scientific, Rockford, IL). For each sample, $20 \mu \mathrm{g}$ total protein or, an equal volume of 20 -fold concentrated media, was loaded and separated on Novex $4 \%$ to $12 \%$ gradient sodium dodecyl sulfate-polyacrylamide gel electrophoresis (SDS-PAGE) gels (Invitrogen, Grand Island, NY). After electroblot transfer onto nitrocellulose membranes and blocking in 5\% nonfat dry milk, membranes were incubated with primary antibodies followed by horse radis peroxidase-conjugated secondary antibodies. Detection was performed using chemiluminescence (Novex ECL; Invitrogen). Specific antibodies used for analysis were rabbit affinity-purified anti-CD44 cytoplasmic tail antisera $(1: 5,000)$ (cytotail antibody) ${ }^{28,29}$ rabbit anti-ITEGE ${ }^{373}(0.5$ $\mu \mathrm{g} / \mathrm{mL}$, generously provided by Dr. Amanda Fosang), ${ }_{16,30,31}$ goat anti-ADAMTS4 (1:1,000, Santa Cruz Biotechnology, Dallas, TX) ${ }^{32}$ rabbit anti-ADAMTS5 $(1: 2,000$, Thermo Fisher, Rockford, IL), ${ }^{32}$ rabbit anti-SMAD1 (1:1,000, Invitrogen) ${ }^{33}$ rabbit anti-phosphorylated-SMAD1/SMAD5/ SMAD8 (1:1,000, Cell Signaling) ${ }^{33}$ and mouse anti- $\beta$-actin (1:10,000, Sigma-Aldrich).

For explant cultures, the cartilage discs were quick frozen with dry ice and ground to a powder in a stainless steel chamber and direct protein extracts were prepared using RIPA buffer (Rockland, Gilbertsville, PA) and Protease Inhibitor Cocktail, followed by the same procedure described above.

\section{Real-Time Reverse Transcription-Polymerase Chain Reaction}

Total RNA was isolated from chondrocyte cultures with TRIzol reagent (Invitrogen), according to the manufacturer's instructions. The RNA was reverse transcribed with qScript cDNASupermix reagents (Quanta BioSciences, Gaithersburg, MD) and amplified at $42{ }^{\circ} \mathrm{C}$ for 30 minutes. For real-time reverse transcription-polymerase chain reaction (RT-PCR), the PCR products were detected using $\mathrm{RT}^{2}$ Real-Time SYBR Green reagents (SABiosciences, Frederick, MD). Primerspecific amplification was performed at $60{ }^{\circ} \mathrm{C}$ for $30 \mathrm{sec}-$ onds. However, fluorescence quantification was performed at a higher temperature $\left(72^{\circ} \mathrm{C}\right)$. The primers pair sequences for bovine genes, forward and reverse, are as follows; GAPDH,
5'-ATTCTGGCAAAGTGGACATCGTCG-3'，5'-ATGGCC TTTCCATTGATGACGAGC-3'; ADAMTS4, 5'-TCACTG ACTTCCTAGACAATGG-3', 5'-ACTGGCGGTCAGCGT CGTAGT-3'; ADAMTS5, 5'-CACCGTGGCTCACGAAA TTG-3', 5'-GGAGCCGAAATTTTCTTCACAGA-3'. All primers were obtained from Integrated DNA Technologies (Coralville, IA). Thermal cycling and fluorescence detection were performed using the SmartCycler System (Cepheid). Real-time PCR efficiencies and the fold increase in copy numbers of messenger RNA (mRNA) were calculated as described previously. ${ }^{34}$ The data are presented as mean \pm standard deviation (SD) by setting negative control as 1.0 , and analyzed by Student's $t$ test. $P$ values $<0.05$ were considered significant.

\section{Results}

\section{Kartogenin Blocks IL-I $\beta$-Mediated Loss of Pericellular Coat Retention on Bovine Articular Chondrocytes}

To examine potential effects of kartogenin on cell-associated pericellular matrices (coats), a particle exclusion assay was used on live bovine articular chondrocytes cultured in monolayer. As shown in Figure 1A and 1B, kartogenin treatment alone did not significantly enhance chondrocyte pericellular coat size. However, the typically observed loss of pericellular coats due to IL-1 $\beta$ treatment (Fig. 1C) was blocked by co-incubation with kartogenin and IL-1 $\beta$ (Fig. 1D). To determine whether kartogenin was affecting biosynthesis and/or assembly of the pericellular coats, kartogenin was added during the regrowth phase, the phase following treatment for 1 hour with 100 units $/ \mathrm{mL}$ testicular hyaluronidase to remove the HA-rich coats. In control chondrocytes, a small coat was reestablished within 24 hours (Fig. 1E). However, in the presence of kartogenin a more robust recovery of the pericellular matrix was observed (Fig. 1F) albeit not as pronounced as the recovery observed in Figure 1D. This suggests that kartogenin may be affecting HA-dependent matrix assembly at multiple levels, in part by promoting synthesis and/or assembly of pericellular matrices.

\section{Kartogenin Blocks IL-I $\beta$-Mediated Loss of Proteoglycan Retention within Neocartilage and Articular Cartilage}

We use bioengineered neocartilages as softer, more manipulatable models of intact adult cartilage. Neocartilages are typically more cellular, rich in HA (and aggrecan) and as such, share similarity to newborn cartilage. A typical, control neocartilage disk is shown in Figure 1G, wherein the tissue exhibited strong safranin $\mathrm{O}$ staining indicative of a HA/proteoglycan-rich extracellular matrix. As with the cell coats, 

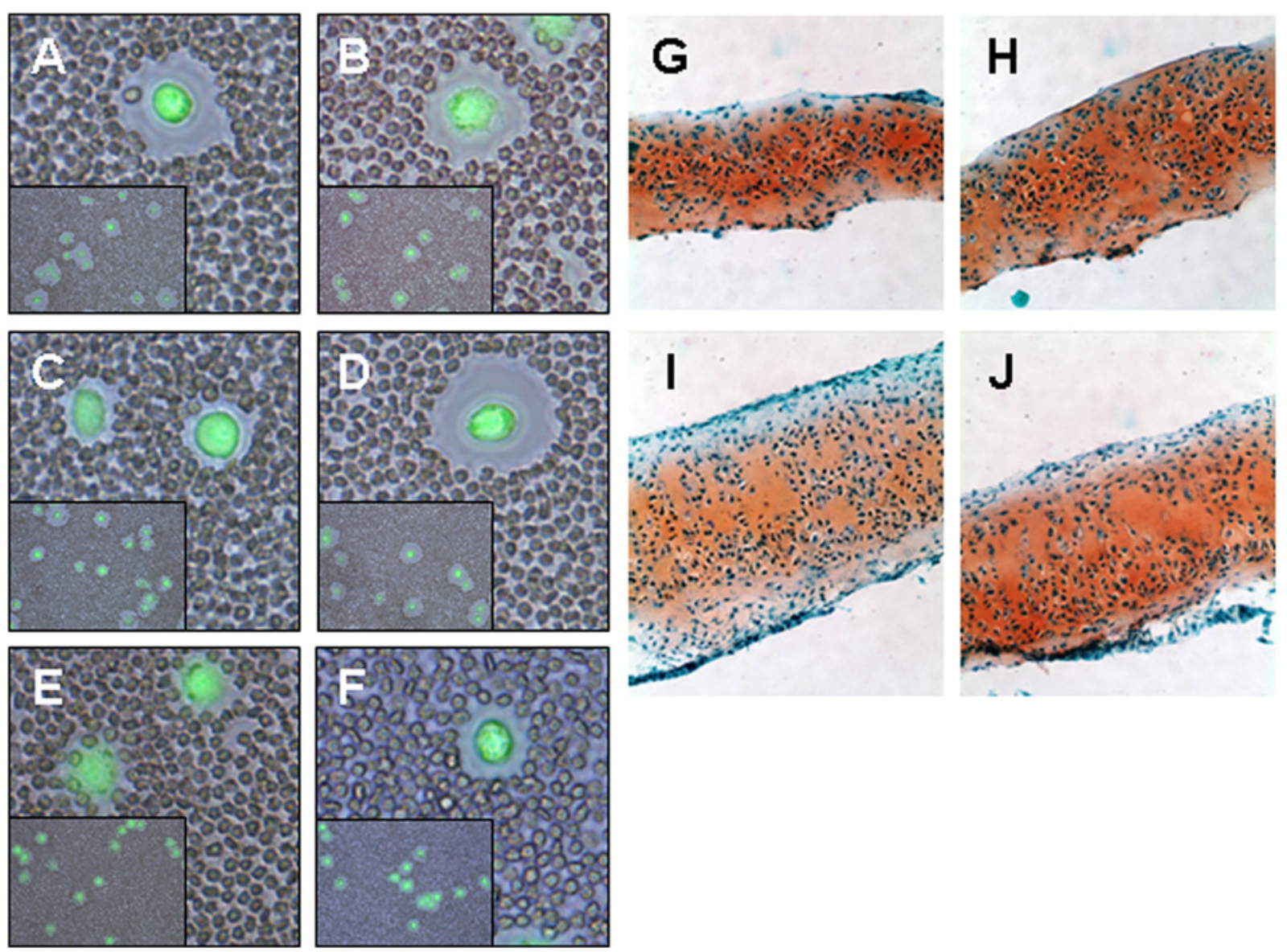

Figure I. Kartogenin blocks IL- I $\beta$-mediated loss of pericellular coat retention on articular chondrocytes as well as loss of proteoglycan retention within neocartilage. Pericellular matrices (coats) were examined on bovine articular chondrocytes cultured in monolayer. Green cells represent live cells labeled with calcein-AM (A-F) and those shown are representative of 2 independent experiments. Chondrocytes were treated with $10 \mathrm{ng} / \mathrm{mL} \mathrm{IL-I} \beta$ in the presence or absence of $10 \mu \mathrm{M}$ kartogenin for 48 hours. Kartogenin treatment alone (B) did not significantly enhance coat size seen on untreated chondrocytes (A). However, the loss of coats due to IL-I $\beta$ treatment (C) was blocked by co-incubation with kartogenin and IL-I $\beta$ (D). If cells were pretreated with testicular hyaluronidase for I hour (E), subsequent kartogenin treatment promoted the recovery of coats $(\mathbf{F})$. Insets in panels A-F depict low power views of each condition. (G-J) Bovine neocartilages were treated with $10 \mathrm{ng} / \mathrm{mL}$ IL-I $\beta$ in the presence or absence of $100 \mu M$ kartogenin for 48 hours. Kartogenin treatment alone $(\mathbf{H})$ did not change safranin $O$ staining, as compared with untreated neocartilages $(\mathbf{G})$; however, the loss of safranin $O$ staining induced by IL-I $\beta$ treatment (I) was inhibited by co-treatment with kartogenin (J).

kartogenin treatment alone (Fig. 1H) did not significantly enhance the safranin $\mathrm{O}$ staining as compared to control neocartilages (Fig. 1G). IL-1 $\beta$ treatment of neocartilage disks resulted in a loss of safranin O staining (Fig. 1I) as expected. The loss of safranin $\mathrm{O}$ staining was blocked with co-treatment of the neocartilage with kartogenin and IL-1 $\beta$ (Fig. 1J).

Full-thickness cartilage discs from bovine articular cartilage cultured as explants exhibited enhanced release of sulfated glycosaminoglycans on IL-1 $\beta$ treatment, as expected, as determined by DMMB analysis ${ }^{26,27}$ of the media. This release of sulfated glycosaminoglycans was significantly reduced with co-treatment of the articular cartilage with kartogenin and IL-1 $\beta$ (Fig. 2).

\section{Kartogenin Treatment Reduces IL-I $\beta$ Stimulation of the Release of GI-ITEGE and ADAMTS5 into the Media of Chondrocyte Cultures}

To examine the effects of kartogenin on catabolism of aggrecan, human (Fig. 3A) and bovine (Fig. 3B) chondrocyte monolayer cultures were treated with IL- $1 \beta$ in the presence or absence of kartogenin, the media were collected and processed for Western blot analysis with the anti-ITEGE, anti-ADAMTS4 and anti-ADAMTS5 antibodies; 3 representative markers of aggrecanolysis. Little to no G1-ITEGE was observed in the medium unless the chondrocytes were first treated with IL-1 $\beta$ (Fig. 3A and 3B). The enhanced 


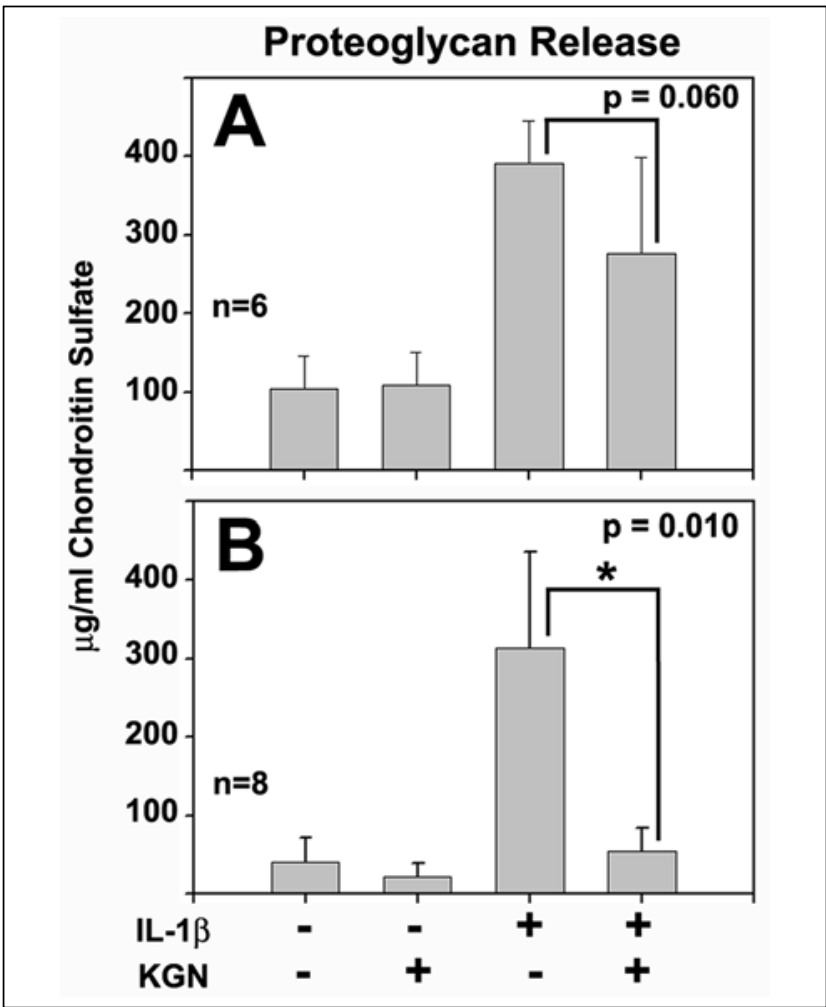

Figure 2. Kartogenin treatment reduces IL-I $\beta$ stimulation of proteoglycan release from articular cartilage explant cultures. Bovine articular cartilage disks were cultured as explants in serum free media the in the absence $(-)$ or presence $(+)$ of $100 \mu \mathrm{M}$ kartogenin (KGN) for 48 hours and then treated and treated without $(-)$ or with $(+) 10 \mathrm{ng} / \mathrm{mL} \mathrm{IL-I} \beta$ for 24 hours. The medium samples were collected and analyzed for sulfated glycosaminoglycans. Data from 6 replicate disks (experiment $\mathbf{A}$ ) and from 8 replicate disks (experiment B) show that KGN treatment alone did not change the release of sulfated glycosaminoglycans from the cartilage explants while IL-I $\beta$ treatment did; however, the enhanced release of sulfated glycosaminoglycans was inhibited by co-treatment with $\mathrm{KGN}$ and IL-I $\beta$. (A, $n=6, P=0.060$; B, $n=8, P=0.010$.)

release of G1-ITEGE was inhibited in cultures co-treated with kartogenin and IL-1 $\beta$ (Fig. 3A and 3B). Similarly, increases in ADAMTS4 and ADAMTS5 (the proteinases that generate G1-ITEGE) were observed only following IL-1 $\beta$ treatment (Fig. 3B). Again, co-incubation of kartogenin with IL-1 $\beta$ blocked the stimulated release of ADAMTS5, and ADAMTS4 to a lesser extent (Fig. 2B). Under the same conditions, the mRNA expression of ADAMTS5 in bovine chondrocytes was elevated following pretreatment with IL-1 $\beta$ (Fig. 2C) and, this increase was blunted by the co-incubation of kartogenin with IL-1 $\beta$ (Fig. 3C). G1-ITEGE accumulation in human osteoarthritic cartilage explants was also examined (Fig. 4B). In the case of these cartilage explants from osteoarthritic patients, no preactivation with IL-1 $\beta$ was required to observe G1-ITEGE

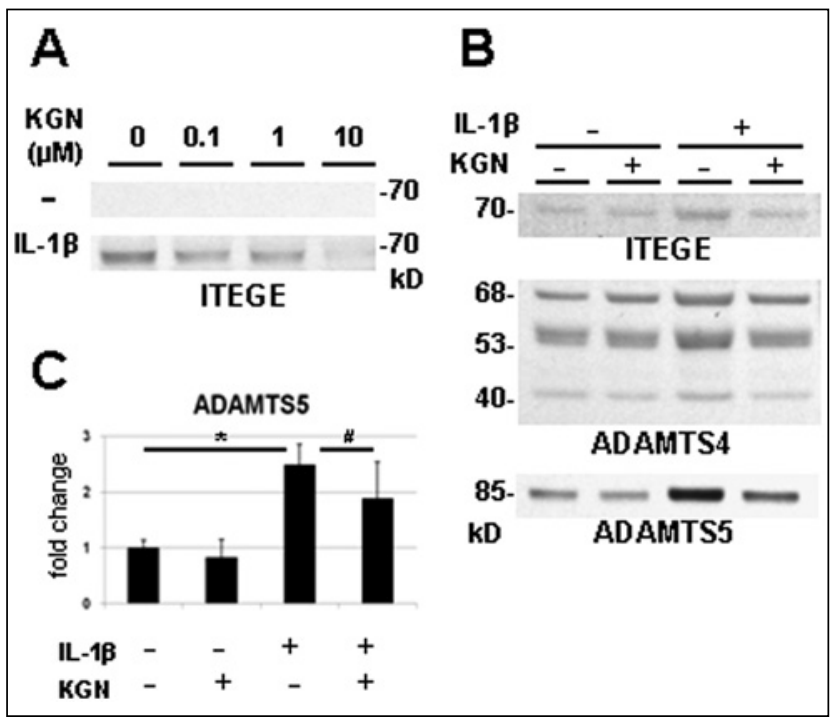

Figure 3. Kartogenin treatment reduces IL-I $\beta$ stimulation of the release of GI-ITEGE and ADAMTS5 into the media of articular chondrocyte cultures. Human articular chondrocytes were cultured in monolayer in the absence $(-)$ or presence $(+)$ of 10 $\mathrm{ng} / \mathrm{ml} \mathrm{IL-I} \beta$ and treated with 0 to $10 \mu \mathrm{M}$ kartogenin (KGN) for 48 hours $(A)$. Bovine articular chondrocytes were cultured with $(+)$ or without $(-)$ I ng/mL IL-I $\beta$ in the presence $(+)$ or absence $(-)$ of $10 \mu \mathrm{M} \mathrm{KGN}$ for 48 hours $(\mathbf{B}, \mathbf{C})$. The media were collected and processed for Western blot analysis (A, B); total RNA was isolated for real-time reverse transcription-polymerase chain reaction (RT-PCR) (C). KGN treatment alone did not stimulate the release of ITEGE while IL-I $\beta$ treatment did; however, the enhanced release of ITEGE was inhibited by co-treatment with $K G N$ and IL-I $\beta$ (A, B). Increasing concentrations of KGN resulted in a proportionate decrease in ITEGE released by IL- I $\beta$-treated human chondrocytes (A). Increased release of ITEGE, ADAMTS4, and ADAMTS5 resulted from the treatment with IL- I $\beta$; however, co-incubation with $\mathrm{KGN}$ blocked the stimulated release of ITEGE and ADAMTS5, while ADAMTS4 did not show significant change (B). Under the same condition, KGN treatment reduced the ILI $\beta$-induced increase of ADAMTS5 gene expression $(C, n=3$; $* P=0.010, \# P=0.033$ ).

epitope by western blot analysis of detergent extracts of the cartilage. Several weeks of treatment of the human osteoarthritic cartilage explants with kartogenin substantially inhibited the accumulation of the G1-ITEGE domains.

\section{Kartogenin Decreased CD44 Fragmentation in Bovine Articular Chondrocytes and in Long-Term Cultured Human Cartilage Explants}

The receptor CD44 plays a critical role in the retention and anchoring of HA and aggrecan. We have shown previously that in association with osteoarthritis, CD44 is cleaved or shed from the surface of chondrocytes leaving the 18 to $20 \mathrm{kD}$ 


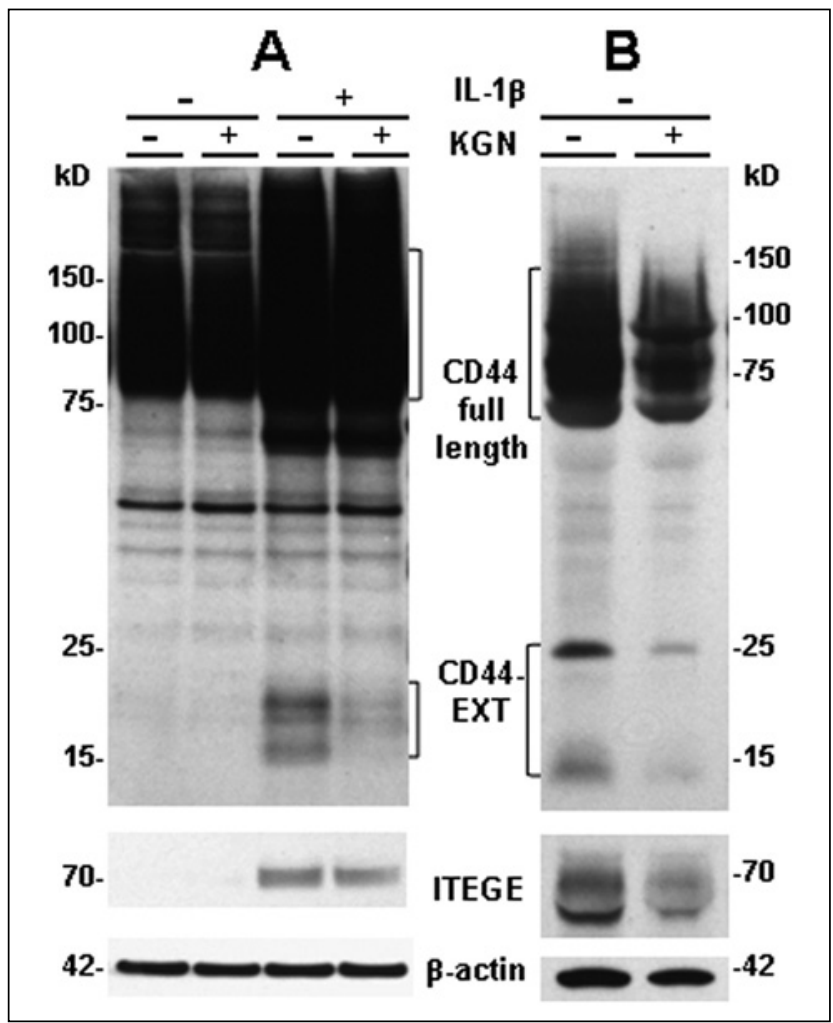

Figure 4. Kartogenin decreases CD44 fragmentation in bovine articular chondrocytes and in a long culture of human osteoarthritis cartilage explants. (A) Bovine articular chondrocytes were stimulated with $10 \mathrm{ng} / \mathrm{mL} \mathrm{IL-I} \beta$ in the presence or absence of $10 \mu \mathrm{M}$ kartogenin (KGN) for 48 hours. Western blot analysis of lysates revealed CD44 expression and fragmentation (CD44EXT) detected with an anti-cytotail antibody and total $\beta$-actin. The IL-I $\beta$-stimulated fragmentation of CD44 was partially blocked when chondrocytes were co-treated with KGN but enhanced total CD44 expression persisted. Western blot analysis of media showed no GI-ITEGE unless the chondrocytes were stimulated with IL-I $\beta$, and the release of GI-ITEGE was reduced on co-treatment with KGN. Blots shown are representative of 2 independent experiments. (B) Human osteoarthritic cartilage was cultured as explants for 6 weeks in the absence or presence of 10 $\mu M K G N$. Western blot analysis of the directly extracted proteins revealed CD44 expression, total $\beta$-actin and total ITEGE. A decrease in the accumulation of GI-ITEGE in the human cartilage treated with KGN was observed accompanied by a decrease of CD44 fragmentation (CD44-EXT).

C-terminal fragment in the membrane (CD44-EXT); an event that we postulate limits the capacity of the receptor to retain HA/aggrecan complexes. ${ }^{29}$ Stimulation of bovine articular chondrocytes with IL-1 $\beta$ resulted in the accumulation of $15 \mathrm{kD}$ and 18 to $20 \mathrm{kD}$ proteolytic fragments of CD44 (Fig. 4A). However, incubation of chondrocytes in the combined presence of IL- $1 \beta$ and kartogenin reduced the production of these CD44 fragments (Fig. 4A). We have also observed similar CD44 fragments in detergent extracts of intact human osteoarthritic cartilage explants cultured in vitro, although the bands are displayed as $15 \mathrm{kD}$ and $25 \mathrm{kD}$ CD44-EXT fragments (Fig. 4B). Parallel cartilage explants treated for several weeks with kartogenin revealed a decrease in the production of CD44 fragments (Fig. 4B). This suggests that the sheddase activity responsible for CD44 proteolytic cleavage is diminished following the application of kartogenin. In the same tissue detergent extracts, the accumulation of G1-ITEGE was explored (Fig. 4B). Again, incubation of the human cartilage explants with kartogenin diminished the accumulation of this end-product of aggrecanase activity suggesting that kartogenin exhibits a generalized anti-catabolic effect.

\section{Kartogenin Stimulates SMAD I Phosphorylation under IL-I $\beta$ Treatment in Chondrocytes}

The effect of kartogenin on the anabolic response of bovine and human chondrocytes was studied by evaluating SMAD1 phosphorylation. As a positive control, BMP7 treatment was used as a means to probe for anabolic responsiveness of our chondrocyte cultures. ${ }^{33}$ BMP7 treatment resulted in phosphorylation of SMAD1/5/8 in both bovine (Fig. 5A) and human (Fig. 5B) chondrocytes as expected. Treatment of these chondrocytes with kartogenin alone resulted in no activation of SMAD1/5/8 suggesting that kartogenin itself cannot directly or via non-canonical cross-talk mechanisms activate SMAD1 (Fig. 5A and 5B). However, when the same concentration of kartogenin was added to bovine or human chondrocytes following IL- $1 \beta$ stimulation, enhanced SMAD1 phosphorylation was observed, notably at the 6 hour timepoint (Fig. 5C and 5D).

\section{Discussion}

During osteoarthritis one of the earliest changes observed is a loss of aggrecan from the extracellular matrix of cartilage. Moreover, this loss is likely initiated within a region near to the surface of chondrocytes, termed the pericellular matrix. Our laboratory has developed techniques to selectively observe and manipulate the glycosaminoglycan-rich pericellular matrix surrounding chondrocytes in vitro. These gellike chondrocyte coats represent hyaluronan/aggrecan/link protein aggregates bound to the cell surface via the hyaluronan receptor, CD $44 .{ }^{35}$ Thus, we reasoned that the retention of these gel coats would represent the first site wherein a predicted chondroprotective effect would ever be observed. The typical loss of pericellular coats due to IL- $1 \beta$ treatment was blocked by co-incubation with kartogenin, while kartogenin treatment alone did not significantly enhance chondrocyte pericellular coat size. In addition, no significant changes in the mRNA expression for chondrocyte-specific genes, such as collagen II, aggrecan, and Sox9, because of kartogenin treatment were observed in either human or bovine articular chondrocytes (data not shown). This suggests that kartogenin exerts more of an effect of blocking 


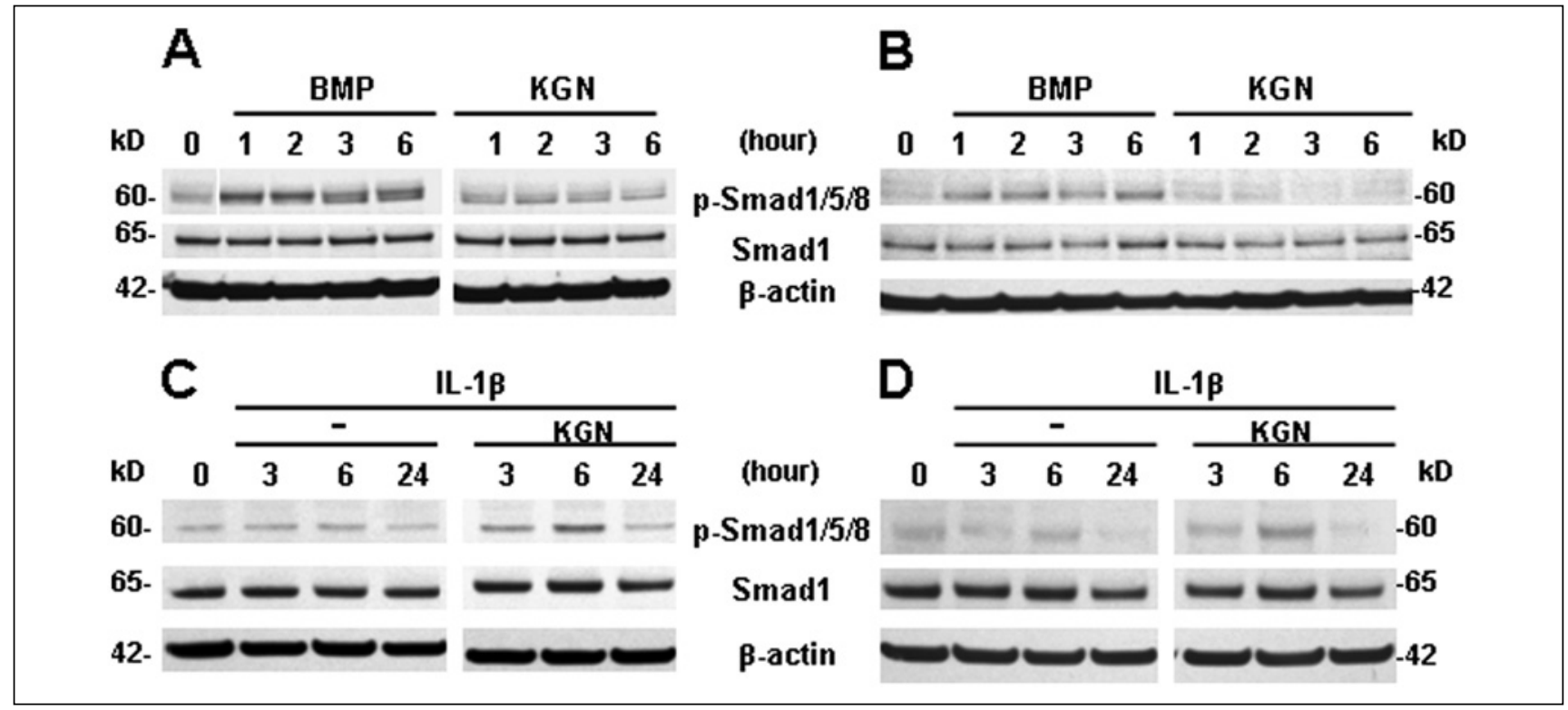

Figure 5. Kartogenin stimulates SMADI phosphorylation only under IL-I $\beta$ treatment in articular chondrocytes. The effect of kartogenin on the anabolic response of bovine and human chondrocytes was studied by western blot analyses of SMADI phosphorylation. Bovine $(\mathbf{A}, \mathbf{C})$ or human (B, D) chondrocytes were treated with $100 \mathrm{ng} / \mathrm{mL}$ BMP7 or $100 \mu \mathrm{M}$ kartogenin (KGN) for I to 6 hours, lysed, and Western blots were analyzed. BMP7 treatment resulted in phosphorylation of SMADI/5/8 as expected, and treatment with KGN resulted in no change in SMADI phosphorylation (A, B). However, following IL-I $\beta$ stimulation for up to 24 hours, KGN treatment resulted in enhanced SMADI phosphorylation, notably at the 6-hour time point (C, D).

degradation than by stimulating repair. Significant blocking effects of kartogenin were also observed on IL-1 $\beta$-induced reduction in safranin $\mathrm{O}$ staining of neocartilage and sulfated glycosaminoglycan release from articular cartilage cultured as explants. Kartogenin blocked the IL-1 $\beta$-mediated loss of proteoglycans in neocartilages and articular cartilage explants as well as but had less effect on stimulating new biosynthesis. Thus, corroborating the original kartogenin report, ${ }^{20}$ we observed a chondroprotective effect, namely blockage of the cellular responses to catabolic stimulation, of kartogenin on chondrocytes and cartilage. On the other hand, little effect was seen by kartogenin treatment alone on non-cytokine-stimulated articular chondrocytes, either of catabolic markers or those related to biosynthesis.

Filamin A, a known actin-binding protein, connects the intracellular filamentous network to cell-membrane proteins, and can sequester core-binding factor $\beta(\mathrm{CBF} \beta)$ in the cytoplasm. ${ }^{36}$ Additionally, hyaluronan-CD44 interactions promote the recruitment of filamin A to lipid raft domains. ${ }^{37}$ Kartogenin is reported to bind to filamin $\mathrm{A}$, displacing $\mathrm{CBF} \beta$ from its cytoplasmic binding site; then $\mathrm{CBF} \beta$ enters the nucleus where it binds to the DNA-binding transcription factor RUNX1. ${ }^{20,40}$ The CBF $\beta-$ RUNX1 complex activates the transcription of proteins involved in cartilage differentiation, increases the synthesis of components of the cartilage matrix (such as collagen type II, aggrecan, and tissue inhibitors of metalloproteinases). This mechanism was proposed for the chondrogenic effect of kartogenin in the original report. ${ }^{20}$
However, on the other hand, $\mathrm{CBF} \beta$ also acts as a co-activator of RUNX2, which is known to play important roles in skeletal development, in terms of hypertrophic differentiation in growth plates, as well as in osteoarthritis pathology. ${ }^{38-42}$ RUNX1 and RUNX2 are regulated differentially, depending on the stage of chondrogenic differentiation. ${ }^{42}$ This might explain why kartogenin does not enhance matrix synthesis in differentiated adult articular chondrocytes.

In the original report, ${ }^{20}$ the chondrogenic effect of kartogenin was demonstrated on primary human mesenchymal stem cells or mouse ATDC5 cell lines, both of which can be induced to differentiate into chondrocytes by growth factor stimulation, including the stimulation by BMPs and other members of the TGFbeta superfamily that can signal through the SMAD transcriptional modulators. Aggrecan, CD44 and HAS-2 genes all exhibit SMAD-binding elements. ${ }^{33}$ The effect of kartogenin on the anabolic response of human and bovine chondrocytes was studied by western blot analyses of SMAD1 phosphorylation. However, the effects of kartogenin on the anabolic response of SMAD1 phosphorylation in response to BMP-7 were only detected on chondrocytes activated by IL- $1 \beta$. These results suggest a possible anabolic effect of kartogenin on catabolicallyactivated chondrocytes.

To investigate the mechanism of the chondroprotective effect of kartogenin, we focused on the degradation of aggrecan. IL-1 $\beta$ stimulated the release of the aggrecan G1 domain fragment domains terminating with the neoepitope 
sequence ITEGE. This ITEGE-containing fragment was reduced following kartogenin treatment of bovine and human articular chondrocytes. Additionally, kartogenin decreased aggrecan G1 domain neoepitope ITEGE accumulation in human cartilage explants. These protective effects on chondrocytes and cartilage appeared to be due to inhibition of ADAMTS5, as shown by reduced levels of ADAMTS5 mRNA as well as released mature protein.

The mechanism whereby kartogenin affects the retention of aggrecan in adult articular cartilage may be mainly by blocking degradation of both CD44 and aggrecan, not by enhanced synthesis. These effects of kartogenin are significant after activation of chondrocytic chondrolysis (e.g., by IL-1, TNF $\alpha$, or oncostatin M). We have shown previously that proteolytic cleavage of CD44 occurs in articular chondrocytes. Moreover, this degradation is pronounced in cells from patients undergoing total knee replacement or in vitro models such as IL-1 exposure, used to mimic osteoarthritis. ${ }^{29}$ This initial cleavage of CD44 occurs by the action of membrane-type metalloproteases such as membrane type I (MT1-MMP), ADAM17 or ADAM10 and could be blocked by inhibitors of matrix metalloproteinases such as GM6001. The residual C-terminal fragment of CD44 (CD44-EXT) then becomes a substrate for intramembranous cleavage by $\gamma$-secretase, releasing an intracellular domain fragment of CD44 into the cytoplasm. In this study, kartogenin blocked the proteolytic cleavage of CD44 suggesting that many of the proteolytic events (MT1-MMP, ADAMTS5, etc.) likely share a common regulatory pathway. Kartogenin treatment was somewhat effective in blocking the IL-1 $\beta$-induced increased expression of ADAMTS-5 mRNA and protein; as well, kartogenin treatment resulted in a reduction in the aggrecan G1-ITEGE neoepitope generated in IL-1 $\beta$ activated cultures. Therefore, likely mechanisms of kartogenin activity include effects on aggrecanases, as well as the proteolytic cleavage of CD44.

In conclusion, these studies provide novel information on the chondroprotective function of kartogenin in adult articular cartilage. This effect of kartogenin is significant after activation of chondrocytic chondrolysis which may occur following disruption of homeostasis maintained by hyaluronan-CD44 interactions.

\section{Authors' Note}

The work reported in this article was done at Department of Anatomy and Cell Biology, The Brody School of Medicine, East Carolina University. The funding sources did not have a role in the collection, analysis, and interpretation of the data.

\section{Acknowledgments and Funding}

The authors thank Joani Zary Oswald and Kayla Britton for technical assistance. The authors acknowledge funding support from the National Institutes of Health (NIH) grants R01-AR043384 and R01-AR039507.

\section{Declaration of Conflicting Interests}

The author(s) declared no potential conflicts of interest with respect to the research, authorship, and/or publication of this article.

\section{Ethical Approval}

This study was approved by our institutional review board.

\section{References}

1. Hardingham TE, Fosang AJ. Proteoglycans: many forms and many functions. FASEB J. 1992;6:861-70.

2. Nishida Y, D'Souza AL, Thonar JMA, Knudson W. IL-1a stimulates hyaluronan metabolism in human articular cartilage. Arthritis Rheum. 2000;43:1315-26.

3. Durigova M, Roughley PJ, Mort JS. Mechanism of proteoglycan aggregate degradation in cartilage stimulated with oncostatin M. Osteoarthritis Cartilage. 2008;16:98-104.

4. Holmes MW, Bayliss MT, Muir H. Hyaluronic acid in human articular cartilage. Age-related changes in content and size. Biochem J. 1988;250:435-41.

5. Pita JC, Muller FJ, Manicourt DH, Buckwalter JA, Ratcliffe A. 1992. Early matrix changes in experimental osteoarthritis and joint disuse atrophy. In: Kuettner KE, Schleyerbach R, Peyron JG and Hascall VC, editors. Articular cartilage and osteoarthritis. New York: Raven Press. p. 455-70.

6. Haapala J, Lammi MJ, Inkinen R, Parkkinen JJ, Agren UM, Arokoski $\mathrm{J}$ et al. Coordinated regulation of hyaluronan and aggrecan content in the articular cartilage of immobilized and exercised dogs. J Rheumatol. 1996;23:1586-93.

7. Morales TI, Hascall VC. Correlated metabolism of proteoglycans and hyaluronic acid in bovine cartilage organ cultures. J Biol Chem. 1988;263:3632-8.

8. Ng KC, Handley CJ, Preston BN, Robinson HC. The extracellular processing and catabolism of hyaluronan in cultured adult articular cartilage explants. Arch Biochem Biophys. 1992;298:70-9.

9. Lark MW, Bayne EK, Flanagan J, Harper CF, Hoerrner LA, Hutchinson NI et al. Aggrecan degradation in human cartilage. Evidence for both matrix metalloproteinase and aggrecanase activity in normal, osteoarthritic, and rheumatoid joints. J Clin Invest. 1997;100:93-106.

10. Sandy JD, Verscharen C. Analysis of aggrecan in human knee cartilage and synovial fluid indicates that aggrecanase (ADAMTS) activity is responsible for the catabolic turnover and loss of whole aggrecan whereas other protease activity is required for C-terminal processing in vivo. Biochem J. 2001;358:615-26.

11. Tortorella MD, Pratta M, Liu RQ, Austin J, Ross OH, Abbaszade I et al. Sites of aggrecan cleavage by recombinant human aggrecanase-1 (ADAMTS-4). J Biol Chem. 2000;275:18566-73.

12. Abbaszade I, Liu RQ, Yang F, Rosenfeld SA, Ross $\mathrm{OH}$, Link JR, et al. Cloning and characterization of ADAMTS11, an aggrecanase from the ADAMTS family. J Biol Chem. 1999;274:23443-50.

13. Glasson SS, Askew R, Sheppard B, Carito B, Blanchet T, Ma HL et al. Deletion of active ADAMTS5 prevents cartilage degradation in a murine model of osteoarthritis. Nature. 2005;434:644-8. 
14. Stanton H, Rogerson FM, East CJ, Golub SB, Lawlor KE, Meeker CT, et al. ADAMTS5 is the major aggrecanase in mouse cartilage in vivo and in vitro. Nature. 2005;434:64852.

15. Embry Flory JJ, Fosang AJ, Knudson W. The accumulation of intracellular ITEGE and DIPEN neoepitopes in bovine articular chondrocytes is mediated by CD44 internalization of hyaluronan. Arthritis Rheum. 2006;54:443-54.

16. Ariyoshi W, Knudson CB, Luo N, Fosang AJ, Knudson W. Internalization of aggrecan G1 domain neoepitope ITEGE in chondrocytes requires CD44. J Biol Chem. 2010;285:3621624.

17. Knudson W, Knudson CB. An update on hyaluronan and CD44 in cartilage. Curr Opin Orthop. 2004;15:369-75.

18. Knudson CB. Hyaluronan receptor-directed assembly of chondrocyte pericellular matrix. J Cell Biol. 1993;120:82534.

19. Nishida Y, Knudson CB, Nietfeld JJ, Margulis A, Knudson W. Antisense inhibition of hyaluronan synthase-2 in human articular chondrocytes inhibits proteoglycan retention and matrix assembly. J Biol Chem. 1999;274:21893-9.

20. Johnson K, Zhu S, Tremblay MS, Payette JN, Wang J, Bouchez LC, et al. A stem cell-based approach to cartilage repair. Science. 2012;336:717-21.

21. Mobasheri A. The future of osteoarthritis therapeutics: emerging biological therapy. Curr Rheumatol Rep. 2013;15:385.

22. Embry J, Knudson W. G1 domain of aggrecan cointernalizes with hyaluronan via a CD44-mediated mechanism in bovine articular chondrocytes. Arthritis Rheum. 2003;48:3431-41.

23. Masuda K, Sah RL, Hejna MJ, Thonar EJ. A novel two-step method for the formation of tissue-engineered cartilage by mature bovine chondrocytes: the alginate-recovered-chondrocyte (ARC) method. J Orthop Res. 2003;21:139-48.

24. Nishida Y, Knudson CB, Knudson W. Osteogenic protein-1 inhibits matrix depletion in a hyaluronan hexasaccharideinduced model of osteoarthritis. Osteoarthritis Cartilage. 2004; 12:374-82.

25. Jiang H, Peterson RS, Wang W, Bartnik E, Knudson CB, Knudson W. A requirement for the CD44 cytoplasmic domain for hyaluronan binding, pericellular matrix assembly, and receptor-mediated endocytosis in COS-7 cells. J Biol Chem. 2002;277:10531-8.

26. Farndale RW, Buttle DJ, Barrett AJ. Improved quantitation and discrimination of sulphated glycosaminoglycans by use of dimethylmethylene blue. Biochim Biophys Acta. 1986;883:173-7.

27. Mort JS, Roughley PJ. Measurement of glycosaminoglycan release from cartilage explants. Methods Mol Med. 2007;135:201-9.
28. Okamoto I, Kawano Y, Matsumoto M, Suga M, Kaibuchi K, Ando M, et al. Regulated CD44 cleavage under the control of protein kinase $\mathrm{C}$, calcium influx, and the Rho family of small G proteins. J Biol Chem. 1999;274:25525-34.

29. Takahashi N, Knudson CB, Thankamony S, Ariyoshi W, Mellor L, Im HJ, et al. Induction of CD44 cleavage in articular chondrocytes. Arthritis Rheum. 2010;62:1338-48.

30. Fosang AJ, Last K, Stanton H, Weeks DB, Campbell IK, Hardingham TE et al. Generation and novel distribution of matrix metalloproteinase-derived aggrecan fragments in porcine cartilage explants. J Biol Chem. 2000;275:33027-37.

31. Mercuri FA, Doege KJ, Arner EC, Pratta MA, Last K, Fosang AJ. Recombinant human aggrecan G1-G2 exhibits native binding properties and substrate specificity for matrix metalloproteinases and aggrecanase. J Biol Chem. 1999;274:32387-95.

32. Ariyoshi W, Takahashi N, Hida D, Knudson CB, Knudson W. Mechanisms involved in enhancement of the expression and function of aggrecanases by hyaluronan oligosaccharides. Arthritis Rheum. 2012;64:187-97.

33. Andhare RA, Takahashi N, Knudson W, Knudson CB. Hyaluronan promotes the chondrocyte response to BMP-7. Osteoarthritis Cartilage. 2009;17:892-902.

34. Ohno S, Im HJ, Knudson CB, Knudson W. Hyaluronan oligosaccharides induce matrix metalloproteinase 13 via transcriptional activation of NFKB and p38 MAP kinase in articular chondrocytes. J Biol Chem. 2006;281:17952-60.

35. Ariyoshi W, Knudson CB, Luo N, Fosang AJ, Knudson W. Internalization of aggrecan G1 domain neoepitope ITEGE in chondrocytes requires CD44. J Biol Chem. 2010;285:36216-24.

36. Yoshida N, Ogata T, Tanabe K, Li S, Nakazato M, Kohu $\mathrm{K}$ et al. Filamin A-bound PEBP $2 \beta / \mathrm{CBF} \beta$ is retained in the cytoplasm and prevented from functioning as a partner of the Runx1 transcription factor. Mol Cell Biol. 2005;25:1003-12.

37. Singleton PA, Mirzapoiazova T, Guo Y, Sammani S, Mambetsariev N, Lennon FE, et al. High-molecular-weight hyaluronan is a novel inhibitor of pulmonary vascular leakiness. Am J Physiol Lung Cell Mol Physiol. 2010;299:L639-51.

38. Marini JC, Forlino A. Replenishing cartilage from endogenous stem cells. N Engl J Med. 2012;366:2522-4.

39. Goldring MB, Tsuchimochi K, Ijiri K. The control of chondrogenesis. J Cell Biochem. 2006;97:33-44.

40. Komori T. Requisite roles of Runx2 and $\mathrm{Cbfb}$ in skeletal development. J Bone Miner Metab. 2003;21:193-7.

41. Kawaguchi H. Endochondral ossification signals in cartilage degradation during osteoarthritis progression in experimental mouse models. Mol Cells. 2008;25:1-6.

42. Kimura A, Inose H, Yano F, Fujita K, Ikeda T, Sato S, et al. Runx1 and Runx2 cooperate during sternal morphogenesis. Development. 2010;137:1159-67. 